\title{
INTERPRETATION DE CERTAINES PROPRIETES DE FILMS GRANULAIRES DE NICKEL
}

\author{
S. MONCHAUD, S. ROLLAND, et R. GRANGER \\ Institut National des Sciences Appliquees, Département de Physique, 35031 - Rennes Cedex, France
}

(Received December 10, 1973, in final form April 22, 1974)

\begin{abstract}
Des films granulaires de nickel (grains de 100 \& environ) sont obtenus par évaporation sur substrat d'alumine en ultra-vide puis recouverts d'une couche d'alumine. La structure et la conductivité électrique de ces grains sont étudiées. La mise en oeuvre de l'analyse par rétrodiffusion d'ions Hélium et les examens au microscope électronique permettent d'estimer les effets d'une oxydation thermique sur ces condensats.
\end{abstract}

\begin{abstract}
Granular nickel films (mean particle size $100 \AA$ ) are obtained through evaporation on alumina substrates under high vacuum then coated with alumina. The structure and conductivity of theses films are studied. An estimation of the oxidation of the nickel particles has been obtained through electron miscroscopy and Helium ion back scattering.
\end{abstract}

\section{INTRODUCTION}

L'étude de la conduction électrique à travers de petites particules métalliques (dimension de l'ordre de $100 \AA$ ) a fait, l'objet d'un grand nombre de travaux tant expérimentaux que théoriques.

Certains concernent la pulvérisation simultanée de petites particules métalliques et d'isolant ${ }^{1,2}$. D'autres se rapportent à des films granulaires discontinus ${ }^{3,4}$ déposés sur substrat diélectrique ionique ${ }^{5,6,7}$ ou isolant ${ }^{8,9}$.

Néanmoins la dégradation rapide de ces films, résultat de mécanismes complexes de pollution et d'oxydation rend délicate l'interprétation des résultats expérimentaux.

C'est pourquoi nous avons réalisé plusieurs configurations constituées à partir de particules de nickel recouvertes d'alumine.

\section{TECHNIQUES EXPERIMENTALES}

\subsection{Preparation des Echantillons}

Les films métalliques granulaires étudiés sont constitués de particules de nickel déposées par évaporation sur un substrat d'alumine. Pour assurer l'homogénéité du milieu dans lequel se trouvent les particules et éviter une pollution de la surface, on les recouvre d'une couche d'alumine.

Cette configuration n'a, à notre connaissance, jamais été étudiée. Les échantillons sont réalisés dans un banc d'évaporation à ultra-vide ULTEK à pompage ionique et sublimation de titane; le vide atteint est de l'ordre de $10^{-8}$ Torr en fin d'évaporation; les évaporations sont réalisées, à température ambiante, à l'aide d'un canon à électrons à quatre creusets qui permet une succession d'opérations sans ouverture de l'enceinte.

Le substrat est une plaquette de corindon, ou une couche épaisse d'alumine ou encore une couche d'aluminium oxydé.

On réalise les contacts électriques par évaporation d'or sur le substrat, puis on dépose entre ces contacts, à température ambiante, les particules de nickel.

Pour permettre une étude de la couche granulaire au microscope électronique, une grille de carbone est placée dans l'enceinte à ultravide à côté du substrat d'alumine. Les matériaux utilisés pour la réalisation des échantillons sont de pureté $5 \mathrm{~N}$.

L'étude électrique est faite sur des échantillons de surface utile $20 \mathrm{~mm}^{2}$, de largeur $5 \mathrm{~mm}$, la distance entre les électrodes est de $4 \mathrm{~mm}$. Pour étudier l'influence de l'oxydation des particules de nickel, certains échantillons on tété chauffés à $270^{\circ} \mathrm{C}$ environ pendant une heure dans un tube en quartz sous un courant d'oxygène humide. Ces échantillons oxydés sont ensuite recouverts d'alumine.

\subsection{Structure et epaisseur des films granulaires}

2.2.1 Contrôle des paramètres d'évaporation L'épaisseur moyenne des couches granulaires de nickel est estimée par la méthode interférentielle, à 
l'aide d'un "Åscope Varian" et par extrapolation de résultats obtenus sur des couches de plus de $100 \AA$ d'épaisseur.

Cette épaisseur moyenne est de $12 \AA$ environ. Cela nous permet simplement de donner un ordre de grandeur de cette épaisseur.

La variation de fréquence d'oscillation d'un quartz vibrant qui reçoit la même épaisseur de matériau évaporé que l'échantillon, nous sert de repère pour assurer la reproductibilité des couches granulaires

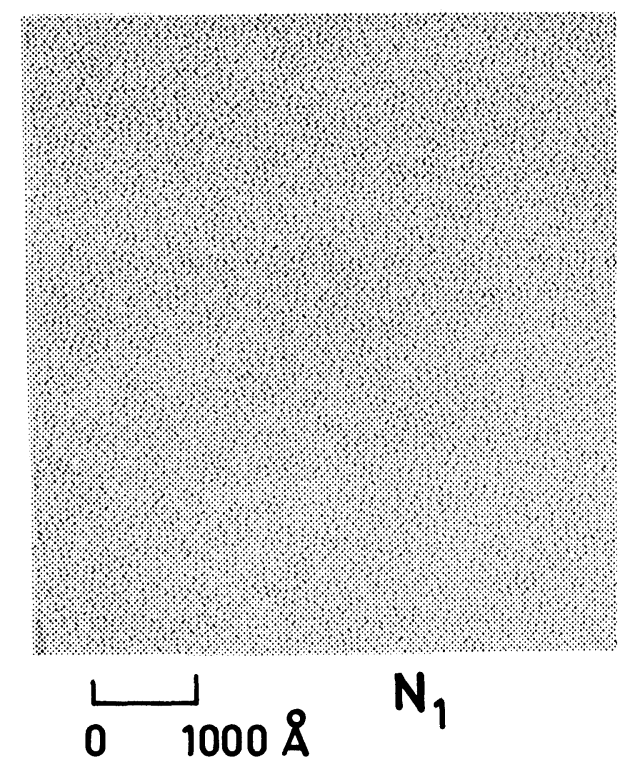

étudiées. Cette variation de fréquence est notée $\Delta f$. La vitesse d'évaporation a été maintenue constante et identique pour tous les échantillons $(\Delta f / \Delta t=20 \mathrm{~Hz}$ minute environ).

\subsubsection{Micrographies électroniques des couches} Les visualisations faites au microscope portent sur les trois types de couches granulaires notées $N_{1}, N_{2}$ ou $\mathrm{N}_{3}$ (Figure 1). Pour étudier une éventuelle influence de la nature du substrat sur la topologie des

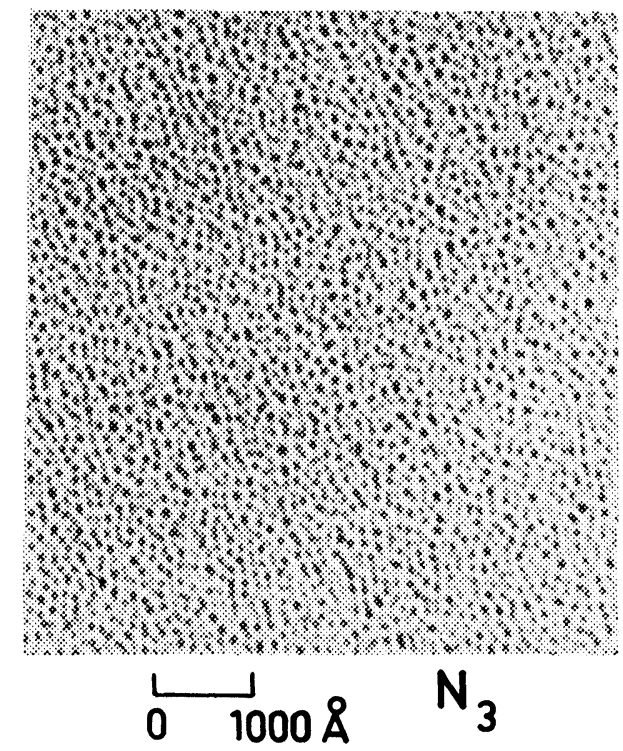

FIGURE 1 Micrographies de couches granulaires de nickel deposées par évaporation sur un substrat de carbone amorphe. 
grains de la couche, des dépôts de nickel de type $\mathrm{N}_{1}$ ont été réalisés sur une plaquette clivée de $\mathrm{NaCl}$ qui a été dissoute avant examen, sur une fine couche d'alumine et sur un substrat de carbone. L'examen des micrographies, obtenues par la technique des répliques, n'a pas révélé de différences appréciables d'aspect des couches ainsi obtenues.

Sur la Figure 1 on constate que la taille des grains croît de $\mathrm{N}_{1}$ à $\mathrm{N}_{3}$ ainsi que la distance entre grains; L'examen des micrographies nous a permis d'évaluer le densité moyenne d'ilôts notée No (Tableau 1).

TABLEAU 1

Estimation de la taille des particules et de la distance entre particules

\begin{tabular}{|c|c|c|c|}
\hline Type de l'échantillon & $\mathrm{N}_{1}$ & $\mathrm{~N}_{2}$ & $\mathrm{~N}_{3}$ \\
\hline $\begin{array}{l}\text { Variation de fréquence du } \\
\quad \text { quartz vibrant } \Delta f(\mathrm{~Hz})\end{array}$ & 80 & 90 & 100 \\
\hline $\begin{array}{l}\text { Densité moyenne d'ilôts } \\
\text { No } 10^{-10}\left(\mathrm{~mm}^{-2}\right)\end{array}$ & 1.56 & 1.06 & 0.61 \\
\hline $2 a(\AA)$ (d'après la formule de Hill) & 76 & 276 & 360 \\
\hline$d(\AA)$ (avec les résultats ci-dessus) & 10 & 18 & 75 \\
\hline $\begin{array}{l}2 a(\AA) \text { (d'après le modèle } \\
\text { d'Hartman) }\end{array}$ & 103 & 272 & 296 \\
\hline
\end{tabular}

On peut constater la bonne concordance des tailles obtenues à partir des deux modèles.

\section{PROPRIETES ELECTRIQUES DES FILMS GRANULAIRES}

Les couches granulaires étudiées ont des résistances très élévées (supérieures au $\mathrm{M} \Omega$ ). On mesure simultanément la tension continue appliquée à l'échantillon et le courant qui circule dans celui-ci par la tension aux bornes d'une résistance en série, le domaine de température exploré est $77^{\circ} \mathrm{K}-300^{\circ} \mathrm{K}$.

Les variations du logarithme népérien de la conductance des couches $(\ln \sigma)$ en fonction de $\sqrt{ } F_{x}$ apparaissent sur la Figure 2 où $F_{x}$ est le champ électrique moyen appliqué dans le plan de la couche.

La conductance est indépendante du champ électrique appliqué jusqu'à $100 \mathrm{~V} / \mathrm{cm}$ pour $\mathrm{N}_{1}, 50 \mathrm{~V} / \mathrm{cm}$ pour $\mathrm{N}_{2}, 20 \mathrm{~V} / \mathrm{cm}$ pour $\mathrm{N}_{3}$. Au-delà de ces valeurs une dépendance linéaire en fonction de $\sqrt{ } F_{x}$ apparaît.

On a étudié la variation de conductance des couches en fonction de la température (cf. Figure 3) pour des valeurs de champ $F_{x}$ inférieures à $20 \mathrm{~V} / \mathrm{cm}$.

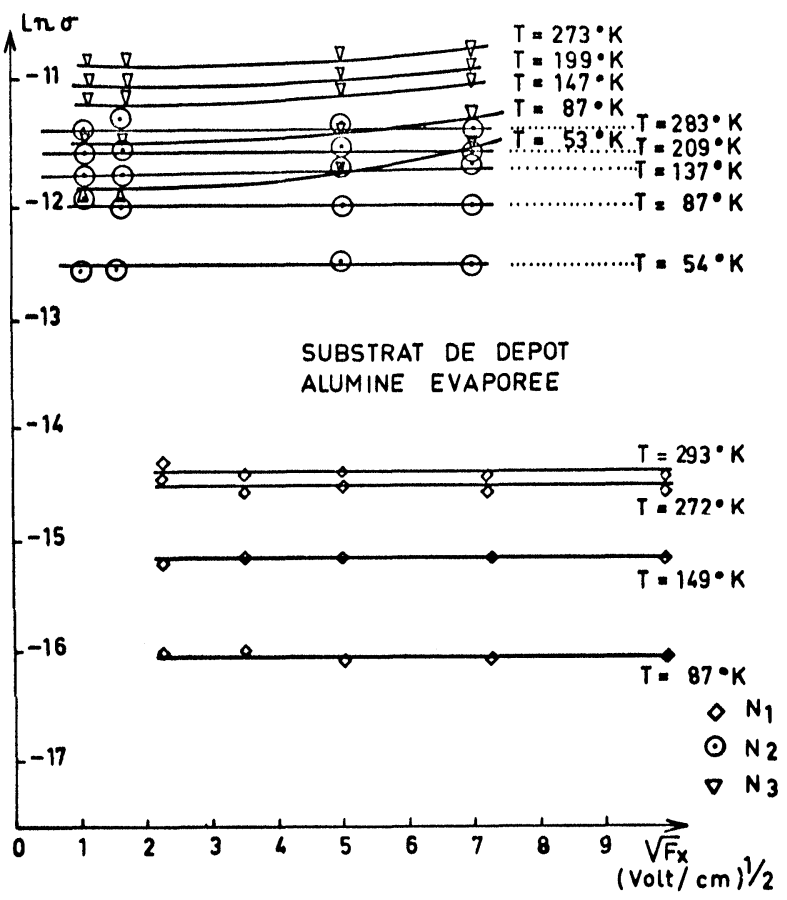

FIGURE 2 Variation du logarithme de la conductance des couches granularres en fonction de $\sqrt{ } F_{x}$ ou $F_{x}$ est le champ électrique moyen appliqué dans le sens de le couche.

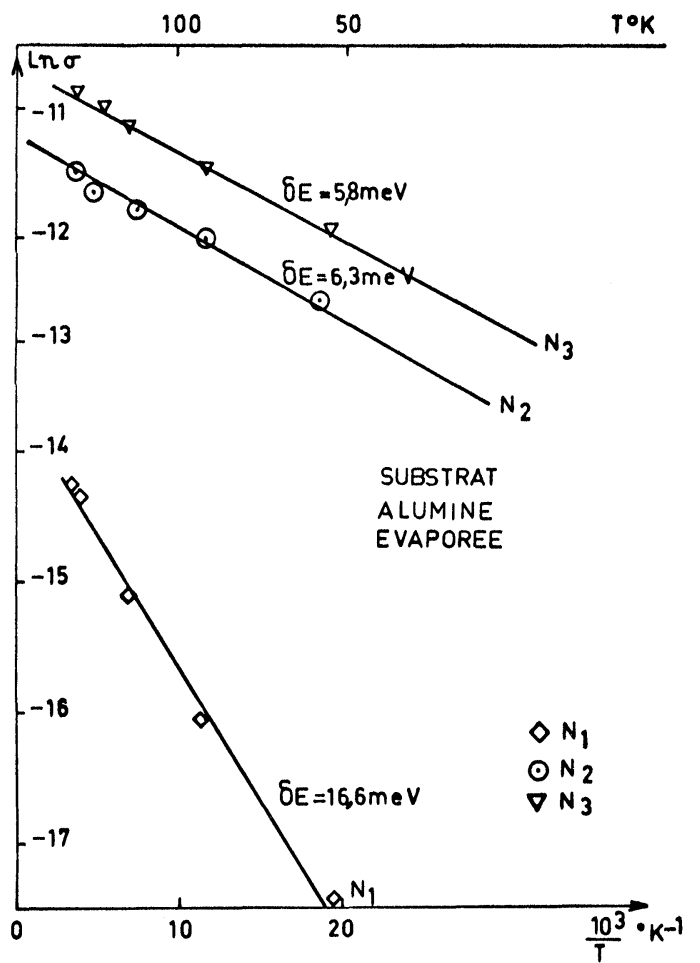

FICIURI: 3 Variation du logarithme de la conductance de coucne, en fonction $1 / T$ où $T$ est la temperature de l'énchantillon. 


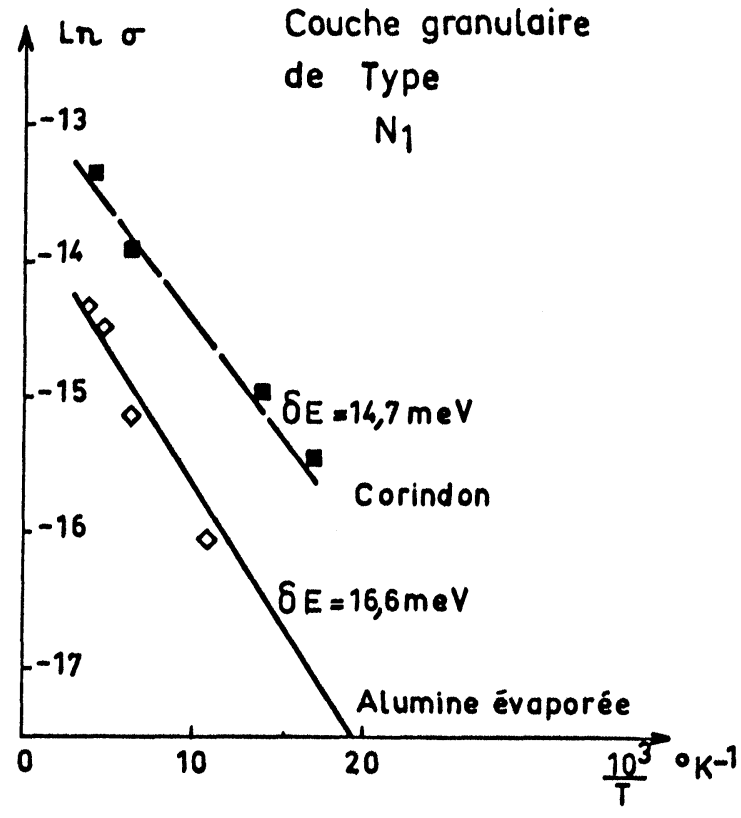

FIGURE 4 Variations de $\log \sigma$ en fonction $1 / T$ pour deux échantillons de type $\mathrm{N}_{1}$

On constate que pour toutes les couches, les variations de $\ln \sigma$ avec $1 / T$ sont linéaires. D'autre part la conductance des couches diminue de $\mathrm{N}_{3}$ à $\mathrm{N}_{1}$.

La Figure 4 montre les variations de $\log \sigma$ en fonction de $1 / T$ pour deux échantillons de type $N_{1}$ préparés l'un sur substrat de corindon, l'autre sur substrat d'alumine évaporée. A champ électrique faible ces variations sont tout à fait comparables.

\section{ESSAIS D'INTERPRETATION}

\subsection{Mise en evidence d'un processus tunnel active entre grains}

Les courbes des Figures 3 et 4 correspondent à une loi de variation de la forme $\log \sigma=-\Delta \phi / K T+$ Cte, ceci caractérise soit une conduction tunnel avec une énergie d'activation $\delta E=\Delta \phi$, soit une conduction thermoélectrique à laquelle correspond le travail d'extraction de l'électron $W=\Delta \phi$.

De la pente des courbes, on déduit $\Delta \phi$

$$
\begin{array}{ll}
\text { Pour } \mathrm{N}_{1} & \Delta \phi=16,6 \mathrm{meV} \\
\text { Pour } \mathrm{N}_{2} & \Delta \phi=6,3 \mathrm{meV} \\
\text { Pour } \mathrm{N}_{3} & \Delta \phi=5,8 \mathrm{meV}
\end{array}
$$

Cette valeur de $\Delta \phi$ est très petite par rapport à la valeur de l'énergie nécessaire pour transférer un électron de nickel dans l'alumine, cette énergie, mesurée expérimentalement, étant de l'ordre de $1 \mathrm{eV}^{10}$. Le mécanisme de conduction apparaît être un processus tunnel activé entre grains avec $\Delta \phi=\delta E$ comme l'a établi Nifontoff ${ }^{1}$.

\subsection{Determination de la distance entre particules et de leur taille}

Les résultats précédents, obtenus à champ électrique faible, permettent de deduire des valeurs de $\delta E$ une valeur moyenne de le taille des particules à l'aide de la formule de $\mathrm{Hill}^{3}$.

$$
\delta E=\frac{e^{2}}{\epsilon_{r} \epsilon_{0}}\left(\frac{(a+d)}{a(2 a+d)}\right)-F_{x} e(2 a+d)
$$

où a est le rayon d'une particule (supposée sphérique); $\epsilon_{0}$ la permitivité diélectrique du vide: $\epsilon_{r}$ la constante diélectrique relative du diélectrique; $F_{x}$ le champ électrique appliqué.

Aux faibles champs $F_{x}$ on peut se limiter au premier terme de cette expression. Hartman ${ }^{9}$ a donné, à partir d'une autre approche l'expression de $\delta E$ :

$$
\delta E=\frac{\hbar^{2} \pi^{2}}{2 m s(2 a) \epsilon_{r}}
$$

où $\mathrm{m}$ est la masse de l'électron; $\epsilon_{r}$ la constante diélectrique relative du diélectrique; $s$ la distance entre les atomes plus proches voisins du matériau constituant un grain; $2 a$ est la dimension moyenne de la particule.

Un film granulaire est assimilé à un ensemble de particules de forme et de taille identiques équiréparties dans un plan. Nous avons choisi la forme hémisphèrique pour représenter les particules parce qu'elle nous paraît une bonne approximation de la forme réelle des particules. L'examen des micrographies conduit à prendre pour configuration celle de l'arrangement compact.

Le motif élémentaire est un triangle équilatéral de côté $(d+2 a)$ où $d$ est la distance entre les particules et $d+2 a$ la distance entre les deux centres de ces mêmes particules. Sa surface est $\sqrt{ } 3(2 a+d)^{2} / 4$. Une particule étant commune à six motifs élémentaires formés chacun de trois particules on peut considérer qu'une demi-particule est associée à chaque motif élémentaire; on en déduit le nombre de particules contenues dans une surface de film $L . l$

$$
N=\frac{L \cdot l}{(\sqrt{ } 3 / 2)(2 a+d)^{2}}
$$


où

$$
2 a+d=\left(\frac{2 L l}{\sqrt{ } 3 N}\right)^{1 / 2}
$$

Le volume total correspondant à ces $N$ particules est $\mathrm{V}=2 \pi \mathrm{a}^{3} \mathrm{~N} / 3$

Les résultats sont présentés dans le Tableau 1 où on a pris pour constante diélectrique de l'alumine la valeur habituelle $\epsilon_{r}=8.8^{12}$ et pour distance entre atomes plus proches voisins du nickel $s=2.49 \AA$.

\section{ETUDE DE L'INFLUENCE DE L'OXYDATION DES PARTICULES DE NICKEL}

Nous avons déposé une couche granulaire de Nickel de type $\mathrm{N}_{1}$, sur un substrat d'alumine. Après oxydation thermique à $270^{\circ} \mathrm{C}$ sous courant d'oxygène humide pendant une heure, les films préalablement recouverts d'alumine présentent une impédance non mesurable avec notre appareillage (supérieure à $100 \mathrm{M} \Omega$ ).

Par suite de la forte diminution du courant tunnel observé dans le domaine de températures de nos experiences $\left(77^{\circ} \mathrm{K}-300^{\circ} \mathrm{K}\right)$, on peut seulement en déduire la conclusion qualitative que la distance entre particules a augmenté après oxydation.

$\mathrm{La}$ diffraction électronique a révélé l'existence d'oxyde de nickel sur des couches de type $\mathrm{N}_{1}$ oxydées (cf. Figure 5).

L'analyse par rétrodiffusion d'ions $\mathrm{H}_{\mathrm{e}}^{+}$a été faite sur une couche granulaire de nickel de type $\mathrm{N}_{1}$ déposé sur un film d'aluminium oxydé dont le support est une plaquette de verre. Cette méthode ${ }^{13}$ consiste à bombarder l'échantillon par des ions $\mathrm{H}_{\mathrm{e}}{ }^{+}$ monocinétiques. La perte d'énergie d'ions rétrodiffusés est caractéristique de la nature des milieux rencontrés par les ions et du trajet parcouru dans ceux-ci. Le nombre d'ions rétrodiffusés augmente avec la masse de matériau rencontré.

La Figure 6 représente le nombre d'ions rétrodiffusés en fonction de leur énergie. La masse de matériau rencontré est proportionelle à l'aire du pic correspondant. L'échantillon non oxydé sert de référence. Après oxydation la courbe en trait plein montre une diminution de la masse de nickel, une augmentation de la masse d'alumine et un déplacement vers le substrat des pics caractéristiques de ces deux matériaux.

Ceci traduit l'oxydation incomplète des particules de nickel et la poursuite de la formation de l'alumine au dépend de l'aluminium.
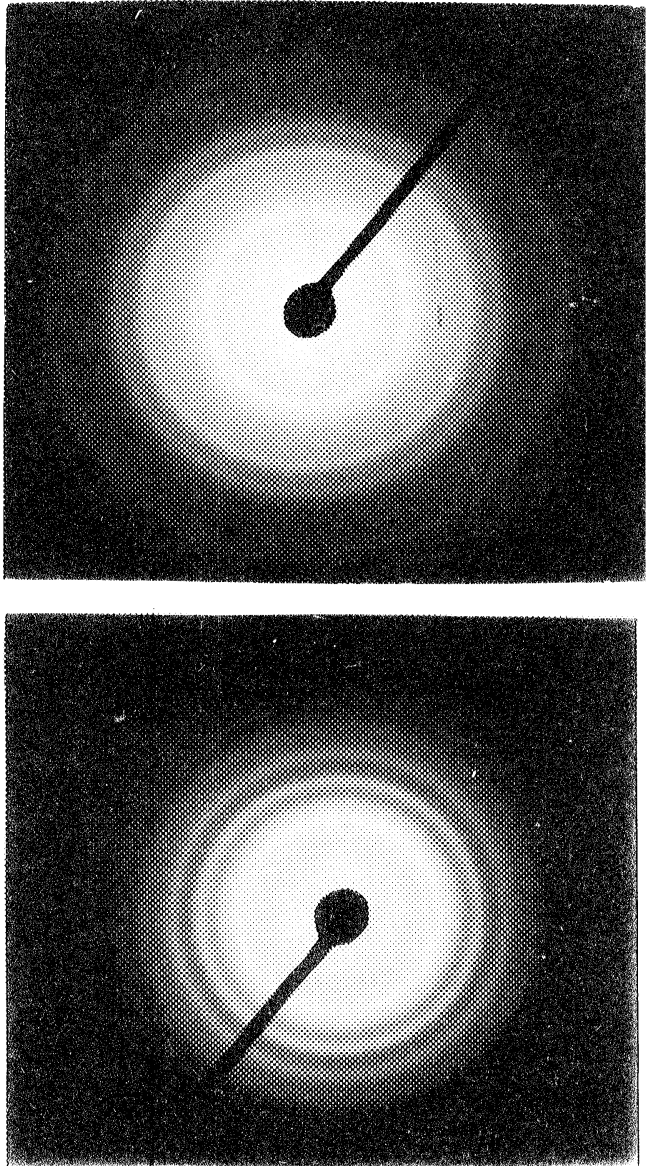

FIGURE 5 Diffraction électronique sur un ensemble de particules de nickel.

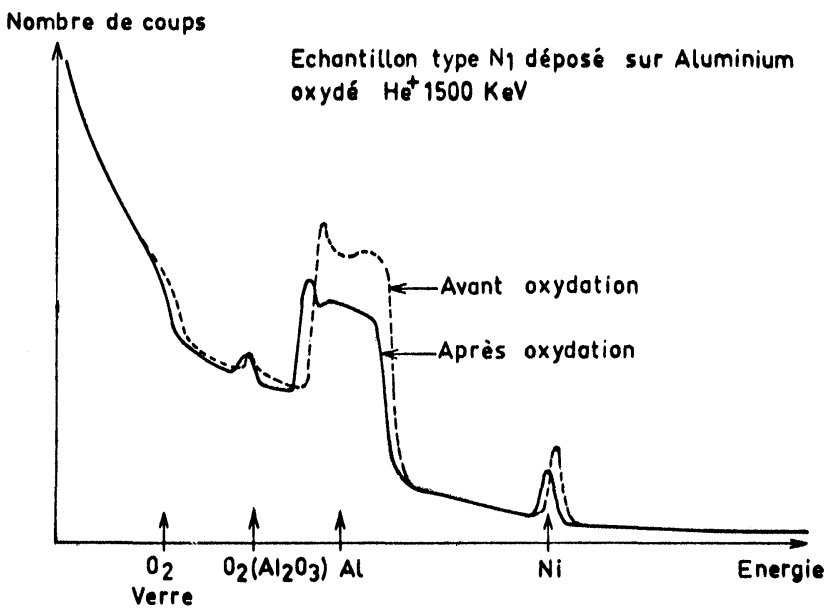

FIGURE 6 Analyse par rétrodiffusion d'ions $\mathrm{He}^{+}$ 
A partir de cette étude, nous pourrions tenter de donner une estimation de la taille des particules et de la distance entre les particules du film oxydé. Supposons que l'arrangement compact décrit bien la topologie des grains après oxydation et que la densité moyenne des grains reste inchangée après oxydation; dans ces conditions $d+2 a$ reste constant pour un échantillon de type $\mathrm{N}_{1}, d+2 a=86 \AA$.

D'autre part le rapport des aires sous les pics caractéristiques du nickel sur la figure après et avant oxydation doit être égal au rapport des masses de nickel libre après et avant oxydation soit $M^{\prime} / M=0,67$ si on pose que $M$ représente la masse de nickel déposé sur la surface de l'échantillon. On tire alors de la formule 3 pour l'échantillon de type $\mathrm{N}_{1}$ oxydé

$\left(\frac{a^{\prime}}{a}\right)^{3} \simeq 0,67$ où $a^{\prime}$ est le nouveau rayon de la particule

d'où

$$
2 a^{\prime} \cong 66 \AA \quad \text { et } \quad d^{\prime} \cong 20 \AA
$$

Ces résultats (en particulier la distance entre particules) ne sauraient expliquer quantitativement la très forte augmentation de résistance de nos films après oxydation. Il est probable que simultanément à l'oxydation il se produit une migration des particules de nickel et peut-être une coalescence ${ }^{15}$. Une étude approfondie serait nécessaire pour donner une interprétation complète du phénomène.

\section{CONCLUSION}

Le but de cette étude était d'étudier la structure de films granulaires de nickel préparés dans des conditions satisfaisantes de reproductibilité. Cette reproductibilité a été assurée par l'utilisation d'une technique originale de préparation des échantillons: évaporation en ultra-vide du film granulaire de nickel sur substrat d'alumine suivie de l'évaporation d'une couche d'alumine. On a pu ainsi éviter la pollution ultérieure et le vieillissement du film granulaire avec le temps. En outre le film se trouve ainsi dans un milieu parfaitement défini. Il faut noter ici que les coefficients de dilation thermique du nickel et de l'alumine sont du même ordre de grandeur et très taibles $\left[\left(\Delta l / l_{0}\right) \cong 10^{-5} \mathrm{~K}^{-1}\right] .{ }^{14}$ De pius ces effets sont d'autant plus faibles que l'adhésion entre le grain métallique et l'isolant qui l'entoure est meilleure ${ }^{3}$, cas dans lequel nous nous sommes efforcés de nous placer. Pour ces deux raisons nous avons négligé l'influence de ces effets.

L'étude de la conduction électrique de ces films noyés dans l'alumine, a mis en évidence une conduction tunnel entre particules avec énergie d'activation. Nous avons pu déterminer expérimentalement cette énergie qui est liée directement à la dimension des particules.

Partant d'un modèle simple nous avons pu estimer la taille moyenne des grains et la distance moyenne entre ceux-ci.

Une analyse par rétrodiffusion d'ions $\mathrm{H}_{\mathrm{e}}{ }^{+}$a permis de suivre le processus d'oxydation thermique des ces films.

Cette méthode d'analyse est à notre connaissance utilisée pour la première fois dans ce genre d'étude.

On a pu en déduire une estimation des effets de l'oxydation sur un film métallique granulaire.

\section{REMERCIEMENTS}

Nous remercions Monsieur Guivarc'h, du Centre National d'Etudes et Télécommunications de Lannion-France, pour l'aide apportée à l'étude structurale de nos échantillons.

\section{BIBLIOGR APHIE}

1. A. Devenyi, R. Manailad and R. M. Hill, Phys. Rev. Lett. 291738 (1972).

2. P. Sheng and B. Abeles, Phys. Rev. Lett. 2834 (1972).

3. R. M. Hill, Proc. R. Soc. A. 309377 (1969).

4. D. S. Herman and T. N. Rhodin, J. Appl. Phys. 371594 (1966).

5. L. Y. Wei, J. Chem. Phys. 392709 (1963).

6. L. S. Palatnik and B. T. Boiko, Sov. Phys. - Dokl. 14 $1211(1970)$

7. B. T. Boiko and L. S. Palatnik, Sov. Phys. - Solid State 121882 (1972).

8. C. A. Neugebauer and M. B. Webb, J. Appl. Phys. 3374 (1962).

9. T. E. Hartman, J. Appl. Phys. 34943 (1963).

10. B. Bouat, Thèse de 3 ème Cycle, Monpellier (1966) Non Publiée.

11. M. N. Nifontoff, Comptes Rendus Acad. Sci. France. 2362486 (1953).

12. K. L. Chopra, Thin Film Phenomena (McGraw-Hill, New York 1969) p. 472.

13. A. Guivarc'h and L. Berthou, Document de Travail $C P M / P M T / 12$ CNET Lannion.

14. R. K. Kirby, American Institute of Physics Handbook (McGraw-Hill, New York 2ème Éd. 1963).

15. J. J. Metois, M. Gauch and A. Masson, Surface Sci. 3043 (1972). 

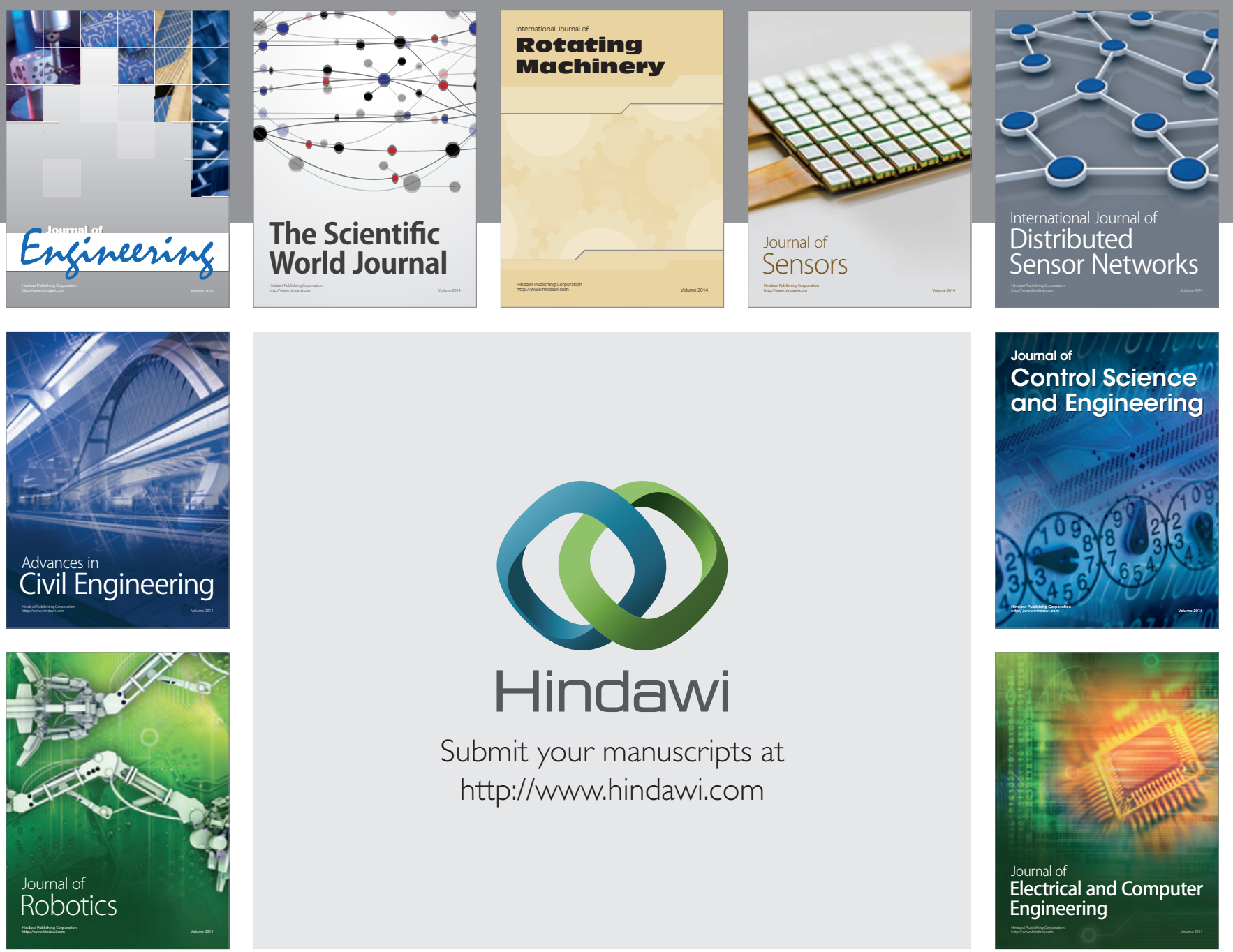

Submit your manuscripts at

http://www.hindawi.com
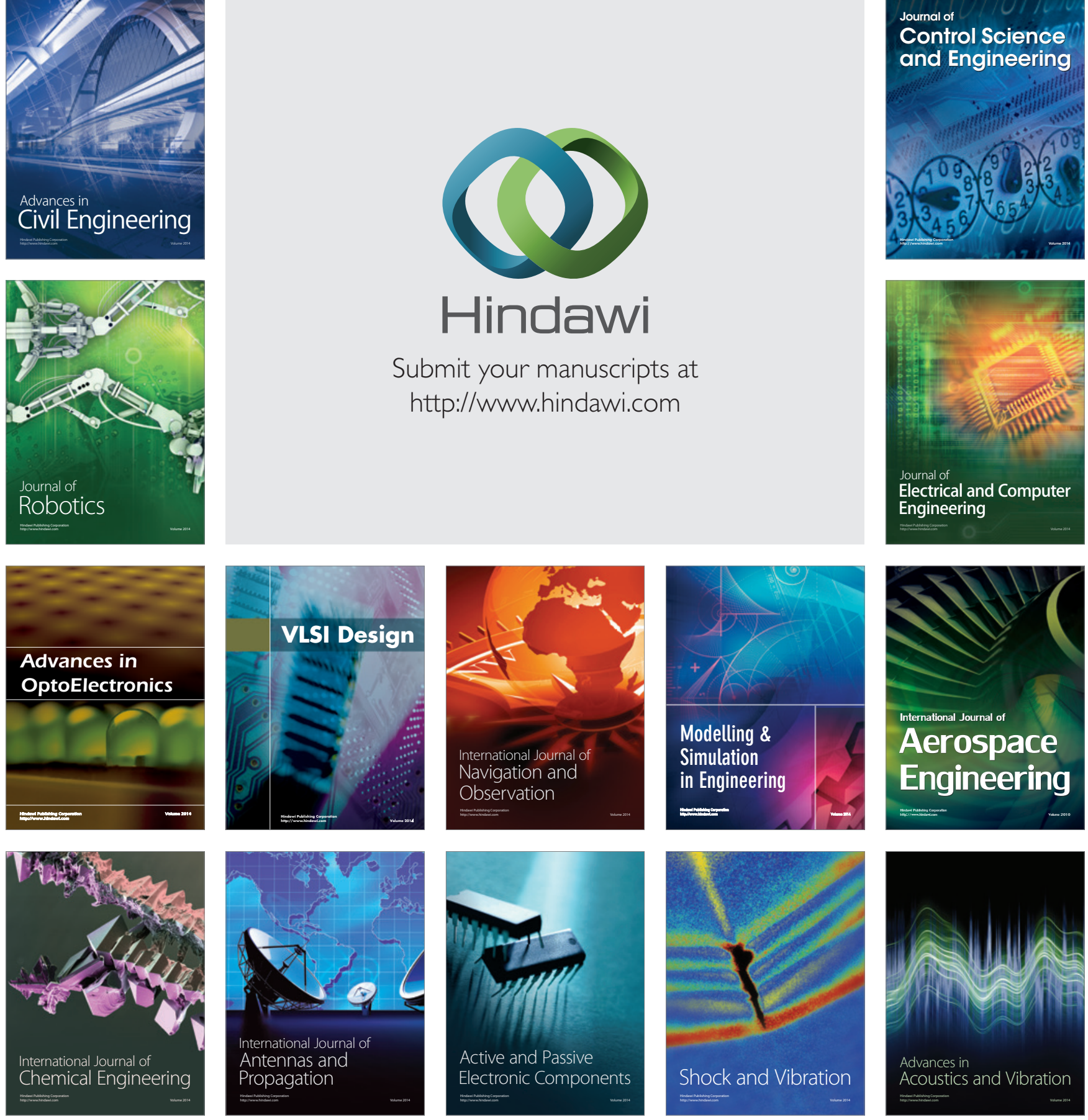